\title{
A Network-Based Asynchronous Architecture for Cryptographic Devices
}

\author{
Kantharaju H $\mathbf{M}^{1}$, Dr. M Srinivas ${ }^{2}$ \\ Selection Grade Lecturer, Department of Computer Science \& Engineering, Govt. (Women's) Polytechnic, \\ Ramanagara, Karnataka, India ${ }^{1}$ \\ Principal and Director Research and Development, St. Mary's Group of Institutions, Hyderabad, India ${ }^{2}$
}

\begin{abstract}
The conventional model of cryptography looks at the security of the figure as a mathematical function. In any case, figures that are secure when determined as mathematical functions are not really secure in genuine usage. The physical usage of figures can be to a great degree hard to control and frequently spill supposed side-channel data. Sidechannel cryptanalysis attacks have appeared to be particularly powerful as a down to earth implies for attacking usage of cryptographic algorithms on straightforward equipment stages, for example, brilliant cards. Foes can acquire delicate data from side-channels, for example, the planning of operations, power utilization and electromagnetic outflows. A portion of the attack techniques require shockingly minimal side-channel data to break a portion of the best known figures. In compelled gadgets, for example, brilliant cards, direct usage of cryptographic algorithms can be broken with insignificant work. Avoiding these attacks has turned into a dynamic and a testing region of research. Control investigation is an effective cryptanalytic technique that concentrates mystery data from cryptographic devices by examining the power devoured amid their operation.
\end{abstract}

Keywords: Asynchronous Architecture, Cryptographic Devices, security, side-channel, data, attacks, powerful, algorithms, operations, power, techniques, effective.

\section{INTRODUCTION}

Cryptography in its standard setting reviews the security of the figure as a logical limit. In addition, it acknowledges that the secret information can be physically secured in painstakingly planned territories and controlled in close, strong computing environments. Regardless, cryptographic systems are executed on real electronic devices that technique transmit and store data [1]. While working, these devices team up with and affect the earth and discharge a particular entirety of data into indicated side-channels. An attacker can exchange off the secret cryptographic enter set away in these devices by observing data that is spilled into side-channels [2]. This kind of cryptanalysis is known as sidechannel examination various techniques for testing cryptographic algorithms in imprisonment have been created. The most definitely comprehended and analyzed systems, differential cryptanalysis and linear cryptanalysis, can mishandle extraordinarily minimal real characteristics in the figure's information sources and yields. Regardless, these techniques examine only a solitary some segment of a cryptosystem's design: the estimation's logical structure [3]. On the other hand, by using side-channel examination the attacker can abuse deficiencies of physical executions, rather than inadequacies of algorithmic parts of a particular cryptosystem. Advancing exploration over the latest ten years (since 1995) has exhibited that the information transmitted by methods for side-channels, for instance, execution time, computational issues [4], control usage and electromagnetic transmissions [5], can ruin to the security of figures.

A huge number of cryptographic devices, by a wide margin most being adroit cards, are used today in a grouping of employments. These cards execute cryptographic computations in perspective of the riddle enter set away in their memories. The goal of an attacker is to expel the puzzle scratch from a sacrificial table safe card with a particular true objective to change its substance, make duplicate cards or play out an unapproved trade. Two general sorts of attacks can be recognized:

1. Invasive attacks are assaults where the sharp card can be rotted, its chip removed, balanced, inspected, mostly squashed or used as a piece of a particular normal setting. These attacks leave discernible affirmation of changing. They usually require a ton of time, current (every now and again uncommonly exorbitant) equipment and unequivocal learning of the card's internals. In view of these factors, prominent attacks are regularly associated with focus information about the astute card structures, and now and again to think information about individual customers. These ambushes join blame assaults and probing attacks [6].

2. Non-obtrusive attacks are ambushes where the sagacious card is inertly checked in the midst of its operation and correspondence with a (possibly balanced) wise card scrutinized. No proof of adjusting is evident from these ambushes. They require inconsequential wander and can be finished in for the most part short measures of time. These 
characteristics of non-invasive attacks have made them of remarkable eagerness for late years. Non-invasive attacks consolidate side-channel attacks and glitch attacks [7]. The focus of this hypothesis is on side-channel attacks specifically.

Side-channel attacks were first found by Paul Kocher in 1995. The chief side station revelation was the timing attack [8] which uses timing information to close the estimations of the riddle keys. This attack misuses shortcomings in executions of the watched cryptosystem, and relates the time anticipated that would play out the cryptographic operation with the operations performed and the information parameters. A typical instance of these weaknesses is branches in the code that depend on upon the estimations of the riddle key, found in square-and-increment algorithm that is used as a piece of figures, for instance, RSA. The accompanying assault to show up, the power analysis attack, was found in 1998 by Paul Kocher and his gathering of experts from Cryptography Research in San Francisco. Kocher et al. depicted two sorts' of attacks: simple power analysis (SPA) and differential power analysis (DPA). Key to these attacks is the observation that the power eaten up by the cryptographic contraption (for this circumstance the sharp card) at a particular time in the midst of the cryptographic operation is related to the bearing being executed and to the data being readied. One of the musings to keep the planning attack on the square-and-copy algorithm was to pad the code with sham figuring's, for instance, exhaust circles Kocher et al. seen that the power usage of these fake estimations was not exactly the same as the power use of huge ones. By simply watching the power takes after obtained from the RSA coprocessor, they could make sense of which operations were performed, what enabled them to reveal the secret illustration? This is the commence of essential power examination. A significantly more powerful attack, the differential power analysis (DPA), relies on upon playing out a true examination of a generous number of encryptions with known plaintexts (or figure compositions). There are varieties of this attack that don't require the learning of either plaintexts or the figure compositions and varieties that use more present day quantifiable procedures, known as higherdemand DPA attacks.

\title{
Objectives of the study:
}

1. To study the Design of the Network-based Asynchronous Architecture.

2. To study the Overview of the network-based architecture.

3. To study Design and Evaluation of the Network-based Asynchronous Architecture.

4. To study the Motivation for using asynchronous architectures for cryptographic devices

5. To study the Side-channel analysis of asynchronous architectures.

\author{
Hypotheses of the study: \\ H1: There is relationship between Design and Evaluation of the Network-based Asynchronous Architecture. \\ H2: A key aspect of the Design of the Network-based Asynchronous Architecture. \\ H3: Overview of the network-based architecture.
}

Motivation for Data Security: As depicted above, data security in current computing systems is a troublesome issue. Network connections and remote document framework administrations, while advantageous; regularly make it feasible for an interloper to access delicate information by trading off just a solitary segment of a vast framework. Due to the trouble of dependably ensuring data, delicate records are frequently not put away on networked computers, making access to them by approved clients awkward and putting them out of the range of helpful framework administrations, for example, reinforcement. As a result, the dread that computer data are not unpleasantly private has prompted a circumstance where customary way of thinking cautions us not to endow our most vital data to our generally modern computers. Subsequently, information assurance framework is indispensable in any organization where ordered and mystery information should be shared and secured at the same time. A few occurrences in the current years epitomize the requirement for a secure cryptographic answer for the issue of shielding information from unapproved get to. The dominant part of these episodes include insiders or framework managers which emphasizes the requirement for a safe information security component that ruins unapproved data burglaries and in addition guarantees that undue power is not left in the hands of workers or overseers.

Cryptographic File Systems: While considering file system security, several viewpoints ought to be considered, for example, confirmation, approval, get to control, secrecy and uprightness. Linux frameworks gives confirmation, approval and get to control administrations utilizing Pluggable Authentication Module (PAM); strategy dialect that characterizes record proprietor and gathering, alongside the proprietor/gathering/world read/compose/execute traits of the document; Posix Access Control Lists (ACL's) that gives more stringent get to control on a for every record premise and so forth. For privacy and respectability administrations, Cryptographic File System (CFS) or Encrypting File System (EFS) must be utilized that gives record encryption/unscrambling alongside uprightness instruments, in a safe, productive and straightforward way to the client. Disseminated cryptographic file system ought to likewise give secure remote access over the Network File System (NFS), record sharing among numerous clients, conceivable use by non-favored clients, immovability, incremental reinforcements and so on. 
Issues with Existing Cryptographic File Systems: Cryptographic file systems introduce an additional layer of indirection at a proper place in the framework that gives the essential cryptographic functionality hence, the primary choice to be taken while planning a Cryptographic File System (CFS) concerns the position of this layer. Encryption administrations by cryptographic file systems can be set at record framework level or gadget layer level. In gadget layer frameworks, for example, Loopback Cryptographic File System (Crypto loop) and Device-Mapper Crypto Target (DMCrypt), encryption/decoding happens at gadget layer in bit space, utilizing Linux piece gadget mapper foundation that gives a nonspecific approach to make virtual layers of square gadgets. These frameworks perform encryption with a solitary key on the whole piece gadget, so record sharing is impractical among different clients. They are additionally not helpful for incremental back-ups, can't be mounted by non-special clients and can't be utilized remotely over NFS. At the file system level, CFS can be executed either in client space or in portion space. Cryptographic File System for UNIX (CFS_Unix) and Encrypted File System (EncFS) are two well-known client space cryptographic document frameworks at record framework level. CFS_Unix is executed as altered NFS server and EncFS utilizing the File System in User-space (FUSE) API. They can be mounted by any client on the framework and does not require any adjustments to the bit so can be effectively versatile. The confinement of these frameworks is their poor execution because of incessant setting switches and information duplicates between client space and portion space. They perform encryption with a solitary key on whole registry, so sharing of individual documents is impractical among various clients. EncFS can be utilized securely over NFS. CFS_Unix is equipped for going about as a remote NFS server, so it can be gotten too remotely without requiring an extra NFS mount. This is, in any case, not prescribed because of security issues with plain content passwords and decoded information being transmitted over the network, and furthermore because of poor execution of CFS. E-Cryptfs is the most prominent portion space CFS, incorporated with the Linux bit since 2.6.19. It utilizes stackable record framework interface approach to present a layer of encryption that can fit over any basic document framework. E-Cryptfs is more productive than existing client space cryptographic file systems, specified previously. It performs encryption on a for each record premise and offers help for document sharing among different clients utilizing Public Key Infrastructure (PKI) bolster. It additionally offers help for file integrity utilizing keyed hashes. It can be utilized remotely on top of organized document frameworks. The restrictions of eCryptfs are that, it can't be ported crosswise over various stages and don't give any choices to non-advantaged clients to mount a file system.

Asynchronous micro pipelines synthesis: Register Transfer Level (RTL) combination show disentangled the timed circuits' plan and permitted outline mechanization driving VLSI advance for over 10 years. Synchronous-toasynchronous directed translation (SADT), we accept, is as critical for asynchronous plan computerization as RTL for synchronous EDA. With RTL configuration commanding the business SADT model is particularly advantageous since (1) it offers bolster for existing determinations and (2) it is effortlessly fused into contemporary outline stream utilizing the best accessible RTL amalgamation motors. The handshake usage and information channel association is there by escaped the creator. Like in RTL it is adaptable through a cell library approach. In spite of known methodologies which utilize HDL for small scale pipeline union, our strategy is not an endeavor to express asynchronous formal models as far as HDL. Our blend stream utilizes an off-the-rack RTL union motor as a front-end to bolster consistent HDL conduct details and an indistinguishable motor from a back-end to offer help for the assortment of net rundown particular organizations utilized by post-union apparatuses in ASIC configuration stream. The principle commitment of RTL model to EDA depends on a partition of streamlining and timing (all consecutive conduct is in a connection between registers, all blend and advancement are just about combinational mists).

RTL show (Fig. 1a) depends on global synchronization and timing presumption (calculations are finished in each phase before the following clock edge). Amid each clock cycle each lock experiences two stages: pass and store. Ace slave flip-flounder association where ace lock is timed by one edge of clock flag and slave hook by the inverse edge keeps the enlist from being straightforward at any given time. Correspondingly to pass and store of hooks dynamic entryways experience: assess and energized (reset). These stages guide to offbeat four-stage handshake conventions where the four stages are information ask for recognize (assess) and ask for recognize reset. (Fig. 1b). Notwithstanding division of streamlining and timing SADT display contributes detachment of set and reset stages: for instance each entryway in Null Convention Logic (NCL) [9] is successive yet can be introduced as combinational - independently in set and reset stages. Accordingly in SADT stream rationale optimization remains isolate from consecutive conduct - the motivation behind why SADT streams can be founded on standard synchronous RTL compilers.

Similarly successive conduct blended in RTL continues as before in a miniaturized scale pipeline. Just its execution is changed from all around synchronized utilizing worldwide planning presumptions to nearby handshake with none or neighborhood timing suppositions. This low-level successive conduct usage is done consequently and does not influence the plan detail. Last usage (and this is the principle distinction from RTL) will give the outcome when it can not at the foreordained time as with synchronous RTL. It will flag the information accessibility and sit tight for the earth to recognize the information receipt to yield the new result. 


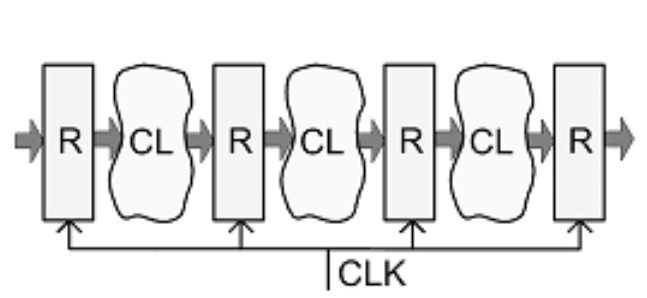

(a) Synchronous pipeline

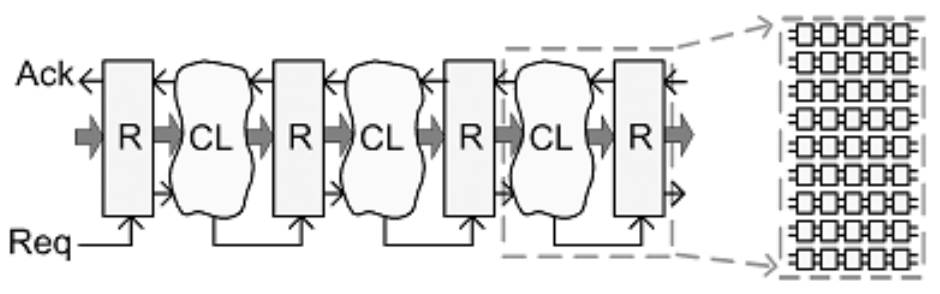

(b) Asynchronous coarse grain

(c) Fine-grain

Fig. 1 Synchronous-Asynchronous Direct Translation: from synchronous (a) to desynchronized (b) and finegrain pipelined (c) circuits

The main distinctive component of our approach is that notwithstanding supplanting global synchronization with nearby self-coordinated control we likewise evacuate practically pointless synchronization and adjust the granularity of pipelining (ordinarily altogether diminish it down to the gate level Fig. 1c). There are a few explanations behind entryway level pipelining: beating parameter varieties, bring down consummation identification overhead especially, we might want to say that lower pipeline granularity is an approach to enhance execution. For security related applications entryway level pipelining permits improvement of little power adjusted doors that can be utilized to consequently blend DPA safe executions. The asynchronous systems (counting handshake correspondence) are avoided the end circuit originator in the miniaturized scale pipeline cell library leaving the handshaking execution to the library designer.

\section{REVIEW OF LITERATURE}

This paper presents a top to bottom writing study of existing cryptographic file systems. The part begins with a portrayal of different plan objectives and outline parameters that ought to be considered while planning a cryptographic file system. A short depiction of different figures and methods of operations utilized by existing cryptographic file systems has been given along a nitty gritty portrayal of XEX-based Tweaked code book mode with figure content Stealing (XTS) [10] that can be utilized by cryptographic file systems for better execution. At that point, existing cryptographic file systems at the square gadget level and at file system level in client space and in bit space are given their focal points and impediments. Facilitate, a concise audit of trusted computing technologies and advantages of utilizing them for key administration in cryptographic file systems has been depicted Finally, Summary of the properties of existing cryptographic file systems has been exhibited alongside the issues distinguished for doing research work.

Cryptographic File Systems Design Goals: A cryptographic file system (CFS) plan may adopt a few strategies concerning the position of encryption layer. The outline of cryptographic file system needs to exchange between adaptability, productivity and security various plan objectives and outline parameters that ought to be viewed as [11] while planning a cryptographic file system are said below:

- Encryption Layer: This decides where the actual encryption/decryption operations are performed on the contents of a file during the write/read process from the disk to memory. This would decide where data remain in plain text during various stages on buffer cache or page cache. The criterion is important because some of these buffers are per-process and others are per-system.

- Transparent access semantics: Encrypted files should support the same access methods available on the underlying file system. All system calls should work in the same way, and it should be possible to compile and execute in a completely encrypted environment.

- Transparent performance: Cryptographic algorithms incur computational overhead, so the performance penalty associated with cryptographic file systems should not be so high that it discourages their use. In particular, interactive response time should not be noticeably degraded.

- Key Granularity and Encryption Target: This refers to the smallest unit which uses the same encryption key. This may be whole file system, a directory or a file. It is also necessary to decide what elements are stored encrypted on the disk, i.e., file data, metadata, file system based information etc.

- Concurrent access: It should be possible for several users (or processes) to have access to the encrypted files simultaneously. Sharing semantics should be similar to those of the underlying file system.

- Protection of network connections: Various attacks like masquerade, interception, replay etc., that may occur to obtain sensitive file data in a networked environment, should be considered.

- Compatibility with underlying system services: Administrators should be able to backup and restore individual encrypted files without the use of special tools and without knowing the key. 
- Portability: A CFS should exploit existing system interfaces for their implementation. Encrypted files should be portable to different operating systems; they should be usable wherever the key is supplied.

- Scale: The encryption engine should not place an unusual load on any shared component of the system. File servers in particular should not be required to perform any special additional processing for clients who require cryptographic protection.

- Limited trust: In general, the user should be required to trust only those components under his or her direct control and whose integrity can be independently verified. A user should not be required to trust the file servers in case of remote file access.

- Compatibility with future technology: Several emerging technologies have potential applicability for protecting data. In particular, keys may be contained in or managed by smart cards or Trusted Platform Module (TPM). Cryptographic file systems should support novel hardware of this sort.

Data Encryption Standard (DES): DES is a block cipher outlined by IBM analysts with help from the National Security Agency (NSA) in the 1970s. It was the primary encryption plot that was embraced as a standard by National Institute of Standards and Technology (NIST) as Federal Information Processing Standard (FIPS) distribution PUB 46. DES utilizes a 56-bit key, a 64-bit piece measure, and can be actualized proficiently in equipment. The overall scheme for DES encryption is outlined in Figure 2. Left-hand side of the Figure shows the preparing of the plain content in three stages first; the 64-bit plaintext goes through an initial permutation (IP) that adjusts the bits to create the permuted input.

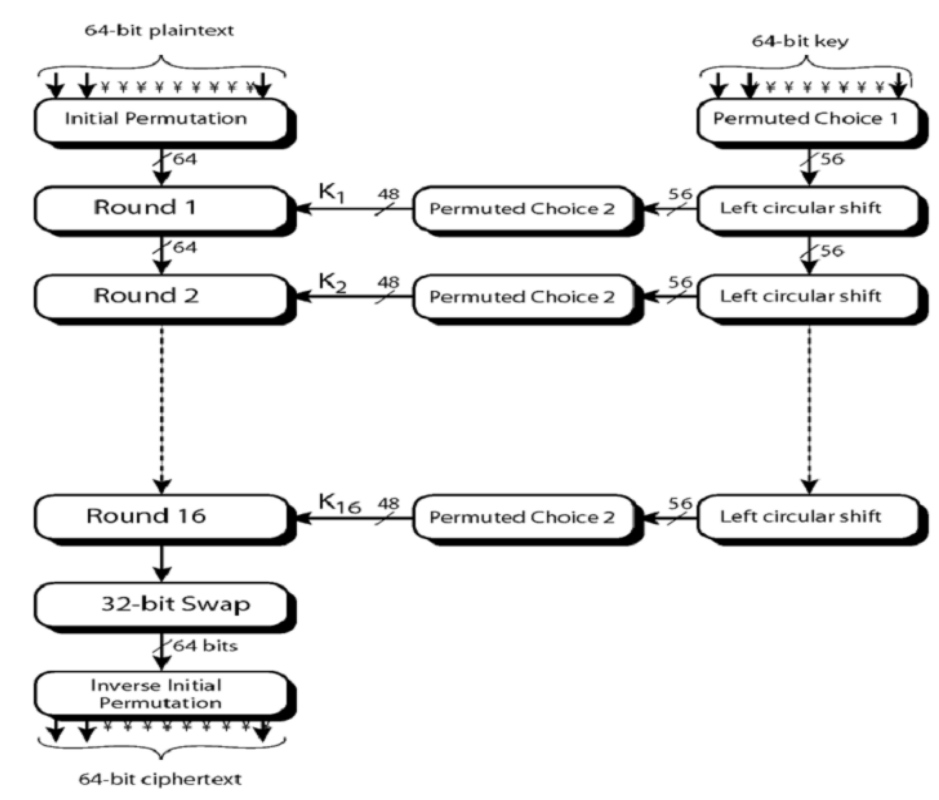

This is trailed by a stage comprising of 16 rounds of a similar capacity, which includes both change and substitution capacities. The yield of the last (sixteenth) round comprises of 64 bits that are an element of the info plain content and the key. The left and right parts of the yield are swapped to create the preoutput. At long last, the preoutput is gone through a stage (IP-1) that is the converse of the underlying change capacity, to create the 64-bit figure content. Except for the underlying and last changes, DES has the correct structure of a Feistel cipher, demonstrated in Figure 3. The right-hand partition of Figure 2 demonstrates the path in which the 56-bit key is utilized. At first, the key is gone through a change work. At that point, for each of the 16 adjusts, a sub key (Ki) is delivered by the blend of a left roundabout move and a stage. The change capacity is the same for each round, yet an alternate sub key is created due to the rehashed movements of the key bits.

DES is no longer considered to be secure due to smaller key size; the brute force attack can break DES in a few hours.

There are a few more secure variations of DES, similar to triple DES. Triple DES FIPS PUB 46 utilizations three separate DES encryptions with three diverse keys, expanding the aggregate key length to 168 bits. DESX is a variation outlined by RSA Data Security that uses a moment 64-bit key for brightening the information before the first round and after the last round of DES, in this manner decreasing its defenselessness to beast constrains assaults, and differential and linear cryptanalysis. 


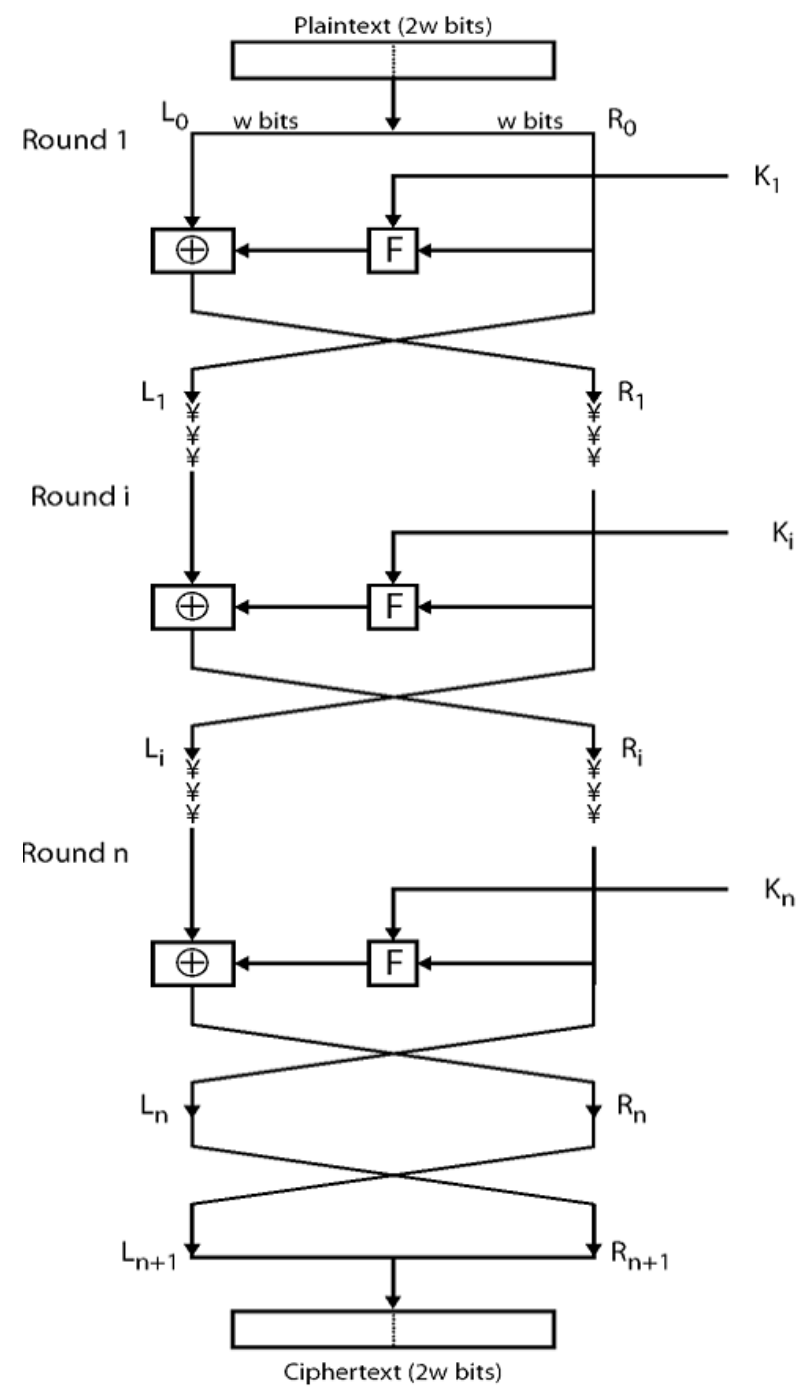

Loopback Cryptographic File System (Crypto loop): Loop back Cryptographic File System (Crypto loop) is most notable file system that performs encryption at the square gadget level. It is a piece of the Linux portions and uses the Linux part cryptographic framework, Crypto API that fares a uniform interface for all ciphers and hashes. The Linux loopback gadget driver shows a record as a piece gadget, alternatively changing the information before it is composed and after it is perused from the native file, to give encryption/decoding. With Crypto loop, the chairman can pick any figure given by Crypto API for file system encryption. The mount bundle on Linux conveyances contains the close-up utility, which can be utilized to set up the Crypto loop.

Crypto loop utilizes the circle gadget as a pseudo gadget that permits each file system calls to be caught for encryption/unscrambling as indicated in Figure 4. This infers all framework and process cradles remain encrypted. Crypto loop encodes the whole file system utilizing a typical mount-time passphrase because of which it has following disadvantages:

- The authentication is solely based on the passphrase and very susceptible to dictionary attacks.

- A single system wide key implies re-encryption of entire file system if one needs to change the key.

- It is impossible for most standard backup utilities to perform incremental backups on the sets of encrypted files without being given access to the unencrypted files.

- No recovery is possible if the user forgets the password as there is no specific recovery agent.

- Sharing a set of encrypted files between different users is not possible.

The remote users will need to use IPSec or some other network encryption layer when accessing the files, which must be exported from the unencrypted mount point on the server. Crypto loop is, however, the best performing cryptographic file system that is freely available and integrated with most GNU/Linux distributions. 

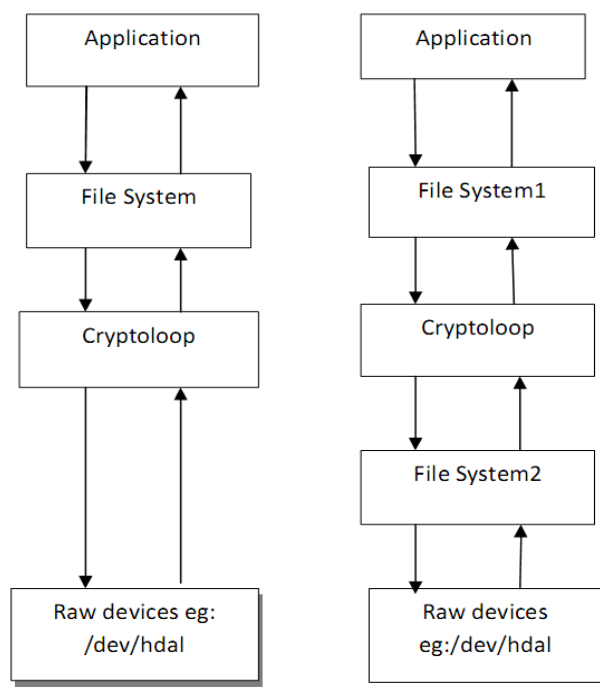

Figure 4: Crypto loop stacked on top of a raw device (left) and a file (right)

Trusted Computing in Cryptographic File Systems: Key management is a fundamental issue in the cryptographic file systems explained in the previous section. The majority of these cryptographic file systems employ only basic password protection schemes, disregarding the best practices of multi-factor authentication. Most passwords that users can reasonably expect to memorize can be successfully attacked with straightforward algorithms running on computing devices in present scenario. Token devices such as smart cards can be used for storing keys in some existing cryptographic file systems; however, the deployment of smart cards has been often prohibitively expensive, cumbersome, and error-prone. Security technology, such as trusted computing can be used for providing multi-factor authentication in a CFS without incurring additional cost. The Trusted Computing Group (TCG) has proposed a Trusted Computing Platform (TCP) based on the Trusted Platform Module (TPM) cryptographic microcontroller system. The TCG is a not-for-profit organization that was formed to develop, define, and promote open standards for hardware-enabled trusted computing and security technologies. The TCP includes both hardware and software components. The functions provided by hardware components are called TPM functions; those provided by the software are called Trusted Software Stack (TSS) functions. The TPM is a hardware chip that enables computers to achieve greater levels of security than was possible earlier. Since the year 2002, most computer manufacturers, including IBM, HP, Dell, Apple, have embedded a TPM chip in the hardware that regulates access to keys based on the state of the machine.

Table 1: Cryptographic file systems

\begin{tabular}{|c|c|c|c|}
\hline Features & $\begin{array}{l}\text { Block-based CFS } \\
\text { (Cryptoloop, } \\
\text { dmCrypt) }\end{array}$ & $\begin{array}{l}\text { User-space CFS at } \\
\text { file system level } \\
\text { (CFS, Encfs) }\end{array}$ & $\begin{array}{l}\text { Kernel-space CFS } \\
\text { at file system level } \\
\text { (eCryptfs, } \\
\text { Transcrypt) }\end{array}$ \\
\hline Performance & Good & Poor & Good \\
\hline Key Granularity & $\begin{array}{l}\text { Common mount-wide } \\
\text { key for whole file } \\
\text { system }\end{array}$ & $\begin{array}{l}\text { Common mount-wide } \\
\text { key for a directory }\end{array}$ & per-file keys \\
\hline $\begin{array}{l}\text { Authentication using } \\
\text { public keys }\end{array}$ & No & No & Yes \\
\hline File sharing support & No & No & Yes \\
\hline Cipher & Crypto API & $\begin{array}{l}\text { DES in CFS, } \\
\text { OpenSSL in Encfs }\end{array}$ & Crypto API \\
\hline Encryption mode & $\mathrm{CBC}$ & $\begin{array}{l}\mathrm{ECB}+\mathrm{OFB} \text { in CFS, } \\
\mathrm{CBC} \text { in Encfs }\end{array}$ & $\mathrm{CBC}$ \\
\hline Integrity Support & No & $\begin{array}{l}\text { Not in CFS, } \\
\text { Supported in Encfs } \\
\text { using keyed hash }\end{array}$ & $\begin{array}{l}\text { Supported using } \\
\text { keyed hash }\end{array}$ \\
\hline $\begin{array}{l}\text { Administrator } \\
\text { Intervention }\end{array}$ & Required & Not required & Required \\
\hline Portability & Not portable & $\begin{array}{l}\text { Portable on any } \\
\text { operating system }\end{array}$ & $\begin{array}{l}\text { Portable on Linux } \\
\text { based systems }\end{array}$ \\
\hline Secure use over NFS & No & $\begin{array}{l}\text { Not in CFS, possible } \\
\text { in Encfs }\end{array}$ & $\begin{array}{l}\text { Not in TransCrypt, } \\
\text { possible in eCryptfs }\end{array}$ \\
\hline TPM Support & Provided in dmCrypt & No & $\begin{array}{l}\text { Provided in } \\
\text { eCryptfs, } \\
\text { Not in TransCrypt }\end{array}$ \\
\hline $\begin{array}{l}\text { Compatibility with } \\
\text { underlying system } \\
\text { services }\end{array}$ & Not Compatible & Compatible & Compatible \\
\hline
\end{tabular}


The TPM therefore provides a hardware-based root of trust and contains the cryptographic functionality to generate, store, and manage cryptographic keys in hardware. The TPM works by measuring the boot loader, kernel, and other critical components of the machine, restricting keys to a strictly defined system state and releasing them only if the machine is booted into its trusted configuration. This approach helps to mitigate attacks that involve booting the machine from unfrosted media. The TSS is the software required to manage the TPM chip. TrouSerS and Trusted Computing for the Java(tm) Platform are the most popular open source TSS packages available. TPM support for key management is provided by dm-Crypt and recent versions of the e-Cryptfs cryptographic file system. As mentioned in Table 1, performance, file sharing, portability and availability to non-privileged users, all cannot be achieved together. Existing user-space cryptographic file systems at file system level have performance limitations and does not provide support for file sharing; and kernel space cryptographic file systems are not portable and cannot be mounted by nonprivileged users.

XTS-AES mode has not been implemented in any of the existing user-space or kernel-space cryptographic file systems at file system level.

The following work has been identified, after a critical analysis of existing cryptographic file systems and their properties:

- Design and implementation of user-space CFS, extending CFS_Unix and EncFS cryptographic file systems, with performance improvements using faster ciphers and file sharing support.

- $\quad$ Design and implementation of secure protocol for CFS_Unix, using cryptographic methods such as mutual authentication and session establishment, which enables its secure use remotely.

- Design and implementation of kernel-space CFS, based on e-Cryptfs, with improved performance using XTSAES and inclusion of whole PKI support in the Linux kernel to exclude privileged user-space processes from domain of trust.

- $\quad$ Use of trusted computing technologies for key management in kernel-space CFS.

\section{CONCLUSION}

This paper presented the outline and assessment of the system constructs asynchronous architecture. It centered in light of investigating a specific equipment worldview for misusing non-deterministic execution so as to profit cryptographic devices. These examinations affirmed that the level of acquainted non-determinism expands resistance with power investigation, when contrasted with straightforward pipelined configurations, as it makes control examination attacks considerably harder to apply. What's more, the non-deterministic execution does not present an execution overhead, in spite of the fact that a tradeoff between the coveted level of the non-determinism (i.e. security) and execution may need to make for a specific usage. This paper introduced a general approach for accomplishing non-deterministic execution in processors. This approach can be corresponding to and joined with existing programming and additionally equipment countermeasures keeping in mind the end goal to accomplish promote imperviousness to side-channel investigation. For example, it could be joined with the randomized booking methods exhibited in further randomize the execution; or it could be actualized utilizing adjusted asynchronous logic as a conceivable approach to shroud the delicate data in the power and electromagnetic follows, which is as yet observed to be available in adjusted frameworks. Electromagnetic attacks are of specific worry as they have turned out to be ready to concentrate data notwithstanding when the power channel gives no data. The non-deterministic and disseminated way of operation of the network-based architecture could conceivably be advantageous against electromagnetic attacks and their capacity to misuse the territory data. Despite the fact that asynchronous circuits have been appeared to be an appropriate stage for abusing non-deterministic execution, a few attributes of asynchrony can be misused in side-channel examination. For instance, information subordinate latencies could be a wellspring of data. One of the points of this approach is to present fine-grain execution timing varieties through randomized steering, which can help diminish this sort of data spillage. The examinations performed in this paper have demonstrated that the presented level of non-determinism impressively convolutes the use of energy investigation assaults and proposes that the presented compositional ideas could be useful to cryptographic devices.

\section{FUTURE WORK}

Future research could continue in various distinctive bearings. A further and more definite assessment of both execution and security features can be performed. In any case, these examinations would require more point by point postponement and power utilization figures, which were not accessible for the present experimentation. Likewise, correlations of the displayed approach could be made with existing techniques for expanding the cost of side-channel analysis attacks. For instance, this approach could be contrasted and the mystery sharing plan. A further investigation of conceivable changes to the design execution could likewise be valuable. Other than investigating routes for 
accomplishing non-deterministic execution, this thesis has exhibited a novel approach for dispersing the control and execution in asynchronous architectures. The non-deterministic execution is accomplished through misusing the finegrain ILP of directions both with and without information conditions. Notwithstanding, the present usage does not actualize any of the conventional techniques for expanding ILP and parallelism all in all. One of the issues that can be researched is the connection of the outline of the network-based architecture to execution enhancing dynamic procedures in chip, for example, theoretical execution, guideline and information stores, theoretical branches and multithreading. When tending to these, a vital issue would likewise be to examine the normal overheads regarding equipment usage. Redress operation within the sight of information conditions frequently requires serialization in the execution of directions, which thusly confines the degree for misusing parallelism. This is especially valid if there should arise an occurrence of RAW conditions, which require the finish of the guideline before its outcome can be utilized by a reliant direction. One of the equipment ways to deal with this issue is information sending. In programming, the impact of genuine conditions could be limited using guideline booking. On account of the networkbased architecture guideline planning could be additionally used to evacuate both asset dispute and slows down in the operand get and branch organize. In particular, if the after effect of the maker direction is as of now accessible when sending solicitations are started, then the guideline execution could be additionally enhanced and slows down decreased. Slows down are additionally undesirable from the security perspective as they can be effectively seen in the power follows. Electromagnetic attacks are much all the more debilitating as they can abuse locality information.

\section{REFERENCES}

[1] S. Chari, C. Jutla, J. Rao, and P. Rohatgi.Template Attacks. In B. S. Kaliski, C, . K. Koc , and C. Paar, editors, Revised Studys from the 4th International Workshop on Cryptographic Hardware and Embedded Systems (CHES 2002), volume 2523-LNCS, pages 13-28. SpringerVerlag, 2002.

[2] Z. C. Yu, S. B. Furber, and L. A. Plana.An Investigation into the Security of Self-timed Circuits.In The Proceedings of the Ninth International Symposium on Asynchronous Circuits and Systems (ASYNC'03), pages 201-210. IEEE Computer Society Press, 2003.

[3] D. Sokolov, J. Murphy, A. Bystrov, and A. Yakovlev. Improving the Security of Dual-Rail Circuits.In M. Joye and J.-J. Quisquater, editors, The Proceedings of the 6th International Workshop on Cryptographic Hardware and Embedded Systems (CHES 2004), volume 3156-LNCS, pages 282-297. Springer-Verlag, 2004.

[4] J. Schneiders. Area Virtual Time.PhD thesis, University of Edinburgh, 2004.

[5] S. S. Salazar. Instruction Scheduling in Micronet-based Asynchronous ILP Processor.PhD thesis, University of Edinburgh, 2002.

[6] R. D. Mullins. Dynamic Instruction Scheduling and Data Forwarding in Asynchronous Superscalar Processors. PhD thesis, University of Edinburgh, 2001.

[7] S. Moore, R. Anderson, R. Mullins, G. Taylor, and J. Fournier. Balanced SelfChecking Asynchronous Logic for Smart Card Applications.Microprocessors and Microsystems, 2003.

[8] T. S. Messerges. Power Analysis Attack Countermeasures and Their Weaknesses.Communications, Electromagnetics, Propagation, and Signal Processing Workshop (CEPS 2000).

[9] D. May, H. L. Muller, and N. P. Smart. Random Register Renaming to Foil DPA. In C, . K. Koc, D. Naccache, and C. Paar, editors, The Proceedings of the Third International Workshop on Cryptographic Hardware and Embedded Systems (CHES 2001), volume 2162-LNCS, pages 28-38. Springer-Verlag, 2001.

[10] A. J. Martin. The Limitations to Delay-Insensitivity in Asynchronous Circuits. In The Proceedings of the 6th MIT Conference on Advanced Research in VLSI, pages 263-278. MIT Press, 1990.

[11] N. Koblitz. Elliptic Curve Cryptosystems. Mathematics and Computation, 48:203-209, 2005. 